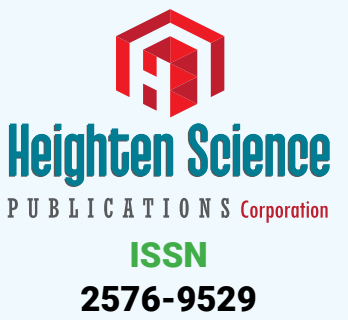

*Address for Correspondence: João Egidio Romão Jr, MD, Hospital BP-Beneficência Portuguesa, São Paulo, Rua Cayowaa, 560 apt 51 05018-000 São Paulo, SP-Brazil, Tel: 55(11)32835304; Email: joao.egidio@uol.com.br

Submitted: 28 October 2017

Approved: 07 November 2017

Published: 08 November 2017

Copyright: @ 2017 Brandão JA. et al. This is an open access article distributed under the Creative Commons Attribution License, which permits unrestricted use, distribution, and reproduction in any medium, provided the original work is properly cited.

Keywords: Acute kidney injury; Hemodialysis; Kidney Disease: Improving global outcomes criteria; Malaria; Outcome
Research Article

\section{The outcome of Acute Kidney Injury in patients with severe Malaria}

\author{
João Alberto Brandão ${ }^{1}$ and João Egidio Romão Jr. ${ }^{2 *}$ \\ ${ }^{1}$ Clínica Multiperfil, Luanda, Angola \\ ${ }^{2}$ Hospital BP-Beneficência Portuguesa, São Paulo, Brazil
}

\section{Abstract}

Background: Acute kidney injury (AKI) is a frequent and serious clinical complication in patients with severe malaria. The purpose of this study was to assess the incidence of AKI in a large population of hospitalized patients with a primary admission diagnosis of malaria, and to investigate the robustness of the KDIGO criteria for predicting the need for dialysis, length of hospital stay and hospital mortality.

Results: We studied 695 consecutive patients admitted with primary diagnoses of malaria, in a 6 months period. AKI occurred in $86(12.4 \%$ ) patients (Stage 1 in $30.2 \%$, Stage 2 in $23.3 \%$ and Stage 3 in $46.5 \%$ ), and 19 (22.1\%) patients required hemodialysis. No patient in the no-AKI or AKI Stage 1 groups (admission or maximum AKI Stage) required hemodialysis, and the requirement of hemodialysis was higher in patients with AKI Stage $2(23.1 \%)$ and Stage $3(42.4 \%)$. The length of hospital stay was longer $(7.3 \pm 7.4$ days vs $5.1 \pm 3.0$ days; $t=4.996$, $\mathrm{p}<0.0001)$, and mortality was higher in patients who developed AKI than in those who did not $(22,5 \%$ vs $2,5 \%$; $\left.x^{2}=79.52 ; p<0.0001\right)$. Patients with AKI Stage 1,2 and 3 had significantly higher hospital mortality $(11 \%, 23 \%$ and $30 \%$, respectively), compared with $2.5 \%$ for patients without AKI [odds ratio $5.2(1.40-19.11, p=0.0331), 13.2$ (4.24-41.06, $\mathrm{p}=0.0002)$, and $16.9(7.26-36.65, \mathrm{p}<0.0001)]$, respectively.

Conclusions: In a relatively large cohort of patients with falciparum malaria infection, the KDIGO criteria identified $12.4 \%$ with a diagnosis of AKI. The KDIGO classification was robust in this population for predicting the need for dialysis, length of hospital stay and hospital mortality. The results support the utilization of the KDIGO criteria in diagnosis and to predicting outcomes for patients with malarial AKI.

\section{Introduction}

Malaria is a major public health problem in the tropical developing world, and it is a major cause of mortality in tropical and subtropical regions [1,2]. The clinical spectrum of renal involvement in falciparum malaria infection varies widely, and acute kidney injury (AKI) is a frequent and serious complication of malaria [3-6].

Recently published studies have used the KDIGO (Kidney Disease: Improving Global Outcomes, 2012) criteria to evaluate the occurrence rate and/or outcome of AKI [7-10]. In countries where immediate access to an ICU and RRT is highly restricted and time consuming, as in sub-Saharan Africa, using criteria of prognosis of AKI can save lives. The aims of this study were to assess the incidence of AKI in patients hospitalized with a primary admission diagnosis of malaria, and the robustness of the KDIGO criteria and their relationship with the length of hospitalization, the need for dialysis and the mortality of these patients during hospitalization.

\section{Patients and Methods}

\section{Study population}

The present study included all adult patients admitted to the Clinica Multiperfil (Luanda-Angola) in a 6 months period. Criteria for inclusion were the occurrence of AKI with positive thick film and/or positive rapid diagnostic test for Plasmodium. 
Patients with chronic kidney disease or on dialysis therapy were excluded, and we only considered the first admission for patients who were readmitted to the hospital during the study period.

The primary outcome variable was AKI defined according to the KDIGO consensus definition [7]. In summary, AKI was defined as an increase in serum creatinine by $\geq 0.3$ $\mathrm{mg} / \mathrm{dL}$ or an increase in serum creatinine to more than 1.5 times at baseline, which is known or presumed to have occurred within the prior 7 days. AKI was staged according to the KDIGO severity criteria, for three levels of injury (Stage 1, serum creatinine levels are increased to more than 1.5-1.9 times than at baseline; Stage 2, more than 2.0-2.9 times than at baseline; and Stage 3, more than 3.0 times than at baseline), upon hospital admission as well as the maximum KDIGO stage reached during hospitalization. The baseline creatinine was estimated by the IDMS-MDRD study equation [11], as recommended by the Acute Dialysis Quality Initiative [8,9]. For analysis, patients were assigned according to their hospital admission and maximum KDIGO category.

We analyzed the demographic characteristics and the evolution of patients during hospitalization: length of hospital stay, need for renal replacement therapy and the outcome (hospital discharge or death). Ethnicity was defined according to the classification upon admission. The diagnosis of malaria was defined by clinical assessment, and was confirmed by blood smear and laboratory testing [12]. Malaria treatment was conducted with clinical support and use of quinine and artesunate derivatives, when indicated. Hemodialysis was performed when indicated and early dialysis was considered in the presence of severe acidosis and/or fluid overload. All data were extracted from electronic medical records, and the procedures were in accordance with the Helsinki Declaration. Informed consent was waived due to the retrospective and noninterventional nature of the study.

\section{Statistical Analysis}

Summary data are presented as mean \pm standard deviation or percentage. Normality of distribution of variables was tested with the Kolmogorov-Smirnov test. Qualitative variables were analyzed using the nonparametric chi-squared or Fisher's exact test, when indicated, and the quantitative normally distributed variables were compared using Student's t parametric test. Univariate and multivariate logistic regression analyses were used to identify risk factors for AKI in malaria at initial presentation, and to determine the association between AKI as defined by KDIGO and the length of hospital stay, need for renal replacement therapy, and hospital mortality. For the variables that were significant in the multivariate analysis. Variables with a $\mathrm{P}$ value $<0.10$ on the univariate analysis were included in the multivariate logistic regression. The Kaplan-Meier curve was constructed to estimate the probability of survival in relation to the presence or absence of AKI and AKI stages. For statistical analysis IBM Statistical Package for the Social Sciences (SPSS) version 22 (IBM Inc., Chicago, IL, USA) was used. A $p$-value of $<0.05$ was considered statistically significant for all comparisons.

\section{Results}

\section{Characteristics of patients with AKI and malaria}

During the study period, 2,172 patients were admitted to the Clinica MultiperfilLuanda, Angola-Table 1. Overall, 706 patients had the primary admission diagnosis of malaria, and 11 (1.5\%) were excluded from the final analysis because they had concomitant diagnoses of chronic renal insufficiency or had been readmitted. Of the remaining 695 patients, 86 (12.4\%) developed AKI. No statistically significant difference was shown in the frequency of malarial AKI between black and white patients ( $\mathrm{p}=0.4264, \mathrm{RR}=1.036,95 \%$ CI 0.9415-1.036); however, AKI was more frequent in Chinese patients than in black $(\mathrm{p}<0.001, \mathrm{RR}=0.1555,95 \%$ CI $1.189-2.033)$ or white 


\begin{tabular}{|c|c|c|c|c|}
\hline & Total & With malaria & $\begin{array}{c}\text { Malaria } \\
\text { - AKI }\end{array}$ & $\begin{array}{c}\text { Malaria } \\
\text { + AKI }\end{array}$ \\
\hline Patients & 2,172 & 706 & 609 & 86 \\
\hline Age $(y)$ & $31.5 \pm 18.6$ & $27.2 \pm 18.5$ & $25.8 \pm 18.9$ & $40.5 \pm 16.1$ \\
\hline Gender (M/F) & $1,129 / 1,043$ & $407 / 299$ & $341 / 279$ & $66 / 20$ \\
\hline $\begin{array}{c}\text { Ethnicity } \\
\text { Black } \\
\text { White } \\
\text { Chinese } \\
\text { CKD/Readmitted }\end{array}$ & $\begin{array}{c}1,898 \\
92 \\
139 \\
43\end{array}$ & $\begin{array}{c}585 \\
38 \\
72 \\
11^{\star}\end{array}$ & $\begin{array}{c}524 \\
28 \\
57\end{array}$ & $\begin{array}{c}61 \\
10 \\
15^{\star}\end{array}$ \\
\hline $\begin{array}{c}\text { Origin } \\
\text { Angolan } \\
\text { Portuguese } \\
\text { Brazilian } \\
\text { Chinese } \\
\text { Other }\end{array}$ & $\begin{array}{c}1,940 \\
43 \\
38 \\
96 \\
55\end{array}$ & $\begin{array}{l}581 \\
24 \\
19 \\
39 \\
43\end{array}$ & $\begin{array}{l}521 \\
17 \\
17 \\
22 \\
43\end{array}$ & $\begin{array}{c}61 \\
8 \\
2 \\
15 \\
0\end{array}$ \\
\hline Need for dialysis & & & & $19(22.1 \%)$ \\
\hline Hospital LOS (days) & $5.4 \pm 5.0$ & $5.4 \pm 3.2$ & $5.1 \pm 3.0$ & $7.3 \pm 7.4^{\star}$ \\
\hline Hospital mortality & $6.4 \%$ & $4.9 \%$ & $2.5 \%$ & $22.1 \% \star$ \\
\hline
\end{tabular}

Results are expressed as mean $\pm S D$; $p$ value estimated using $t$ test, or $x 2$ test. ${ }^{*} \mathrm{p}<0.001$ for malaria-AKI versus malaria+AKI. AKI: acute kidney injury; ${ }^{\star}$ CKD or readmission; LOS: length of stay.

patients ( $\mathrm{p}<0.001, \mathrm{RR}=1.501,95 \%$ CI 1.132-1.989) with malaria. The length of hospital stay was longer in patients who developed AKI than in those who did not $(7.3 \pm 7.4$ days vs. $5.1 \pm 3.0$ days; $\mathrm{t}=4.996, \mathrm{p}<0.0001$ ). Mortality was higher among patients with malaria who developed AKI than among those without AKI $(22.1 \%$ vs. $2.5 \%$; $\chi 2=58.263, \mathrm{p}<0.0001, \mathrm{RR}=95 \%$ CI 1.394-2.976). Among 19 (22.1\%) patients with malarial AKI requiring hemodialysis, the crude mortality was statistically higher than the rate observed in patients with malarial AKI who received conservative treatment ( $47.4 \%$ vs. $16.4 \%$; $\mathrm{p}=0.0011, \mathrm{RR}=2.885,95 \%$ CI $1.406-5.921$ ).

\section{Progression to maximum AKI Stage during hospitalization}

Upon hospital admission, 72 patients had diagnosis of AKI, and they were stratified according to KDIGO criteria: Stage 1 in 26 (36.1\%), Stage 2 in 17 (23.6\%), and Stage 3 in 29 (40.3\%). The progression of AKI of these patients to the maximum AKI Stage after hospital admission is shown in figure 1. During hospitalization, 14 patients without AKI developed AKI, reaching a Stage 1 (11 patients), Stage 2 (1 patient) or Stage 3 ( 2 patients), and 16 patients upon admission classified using KDIGO criteria as Stage 1 and Stage 2 progressed to a more severe AKI stage (Stage 2 and Stage 3, respectively). Overall, AKI stratified according to the maximum KDIGO criteria reached during hospitalization of 86 patients was 27 (30.2\%), 19 (23.3\%) and 40 (46.5\%) for Stages 1,2 and 3, respectively.

\section{KDIGO criteria and the need for renal replacement therapy}

Nineteen (22.1\%) patients with malarial AKI required hemodialysis. In the subgroup of 72 patients with AKI detected at the time of admission, no patient in KDIGO no-AKI or AKI Stage 1 required hemodialysis during their hospital stay; furthermore, this was similarly shown for all patients with maximum AKI Stage 1 during hospitalization. The percentage of AKI Stage 2 patients that required hemodialysis was $23.1 \%$, while this percentage increased to $42.4 \%$ in patients with AKI Stage 3-Figure 2. Statistically significant differences were observed when comparing the requirement for hemodialysis in patients who reached maximum AKI Stage 2 and 3 with the group of patients in AKI Stage 1 and no-AKI $\left(\chi^{2}=253.6 ; p<0.0001\right)$.

\section{KDIGO criteria and length of hospital stay}

The mean length of hospital stay for the 86 patients with AKI was $7.3 \pm 7.4$ days, ranging from 1 to 56 days, and it was longer $(t=4.996, p<0.0001)$ than the length of hospital stay for patients with malaria and who did not develop AKI (5.1 \pm 3.0 days). 
The length of hospital stay for each of the AKI Stage 1, 2, and 3 were 6.1 \pm 4.1 days, $6.5 \pm 6.6$ days and $9.0 \pm 9.7$ days, respectively, and there were no statistically significant differences among the stages $(\mathrm{p}=0.250)$ Figure 2 .

\section{AKI stages and mortality}

Increasing severity of AKI Stage was associated with a higher patient mortality. Patients with AKI Stage 1, 2, and 3 had significantly higher unadjusted hazard ratios for hospital mortality (11\%, 23\% and 30\%, respectively), compared with $2.5 \%$ for malaria patients without AKI $\left(\chi^{2}=79.52 ; \mathrm{p}<0.0001\right)$ - Figure 2. The unadjusted odds ratios (95\% confidence interval) for hospital mortality for AKI and KDIGO stage 1, stage 2, and stage 3 were, respectively, 5.2 (1.40-19.11, p=0.0331), 13.2 (4.24-41.06, $\mathrm{p}=0.0002)$, and 16.9 (7.26-36.65, p<0.0001). Increasing severity of acute kidney injury was associated with higher mortality, notably AKI Stage 2 and AKI Stage 3 had a higher mortality following the AKI episode (log-rank test: $\chi^{2}=32.54 ; p<0.0001$ ) Figure 3.

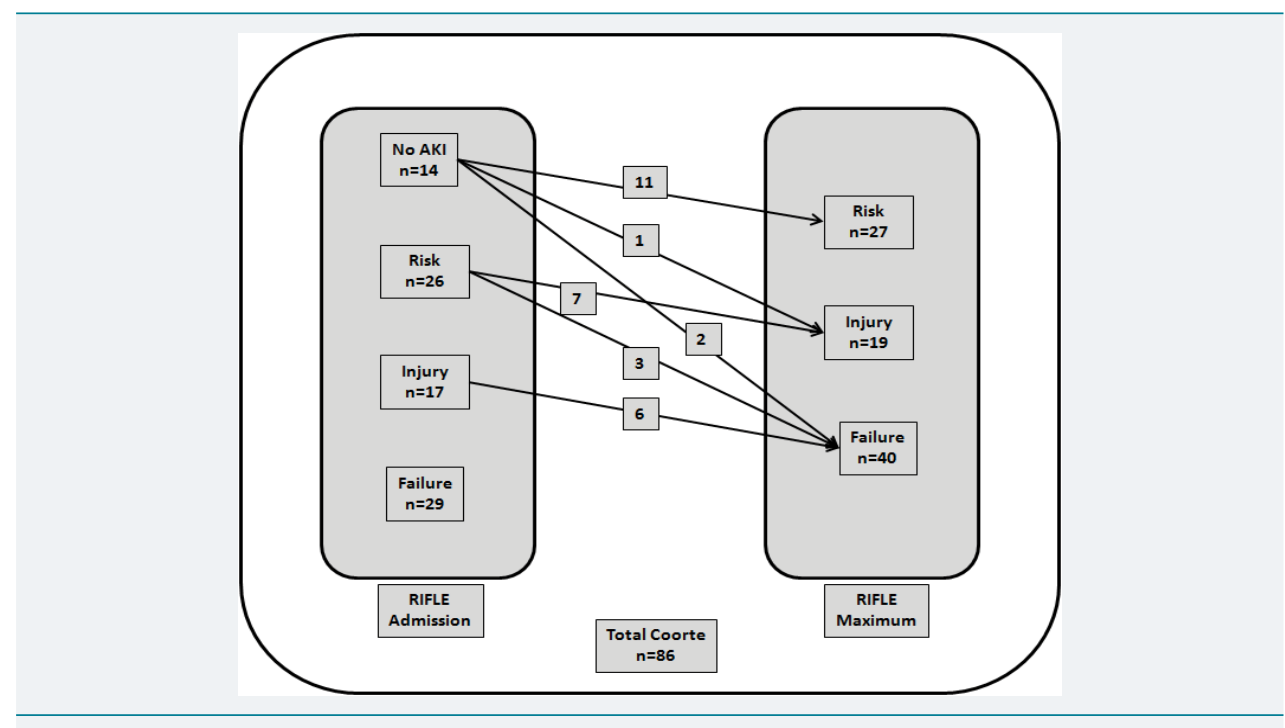

Figure 1: Flow chart of the clinical evolution of 86 patients with AKI until the maximum KDIGO criteria.

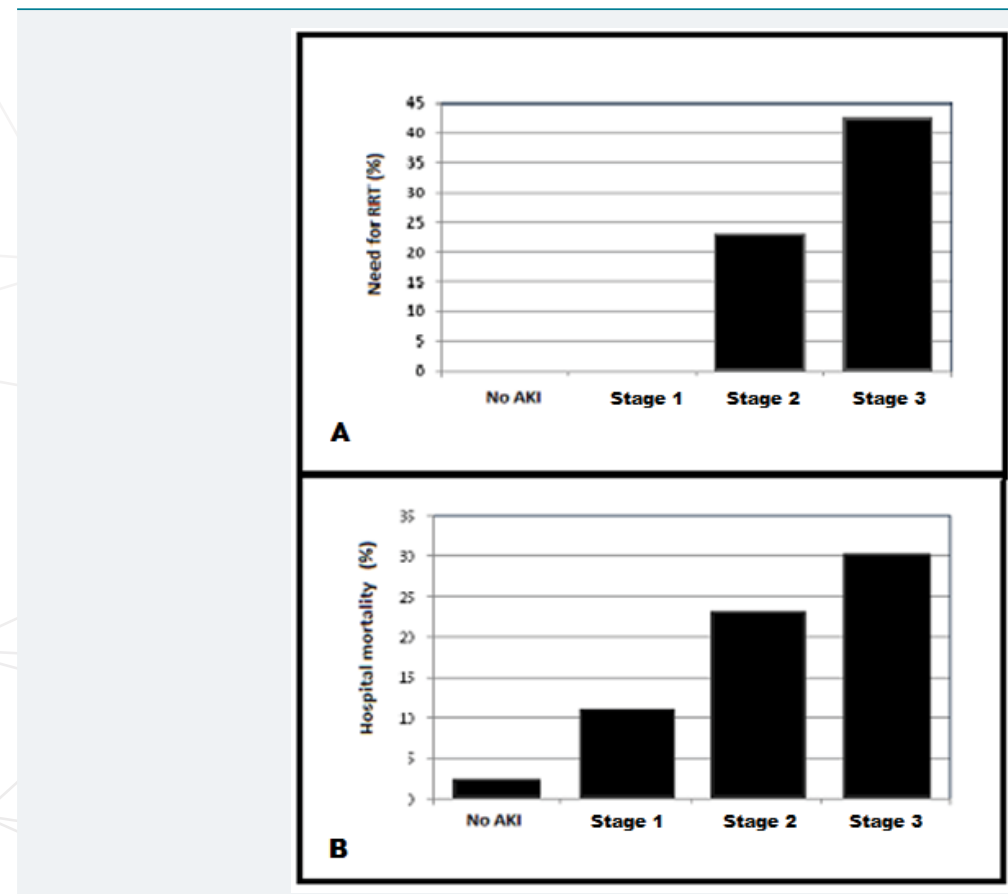

Figure 2: (A) Need for renal replacement therapy (RRT), and (B) hospital mortality among 86 patients with AKI defined by the KDIGO criteria. 


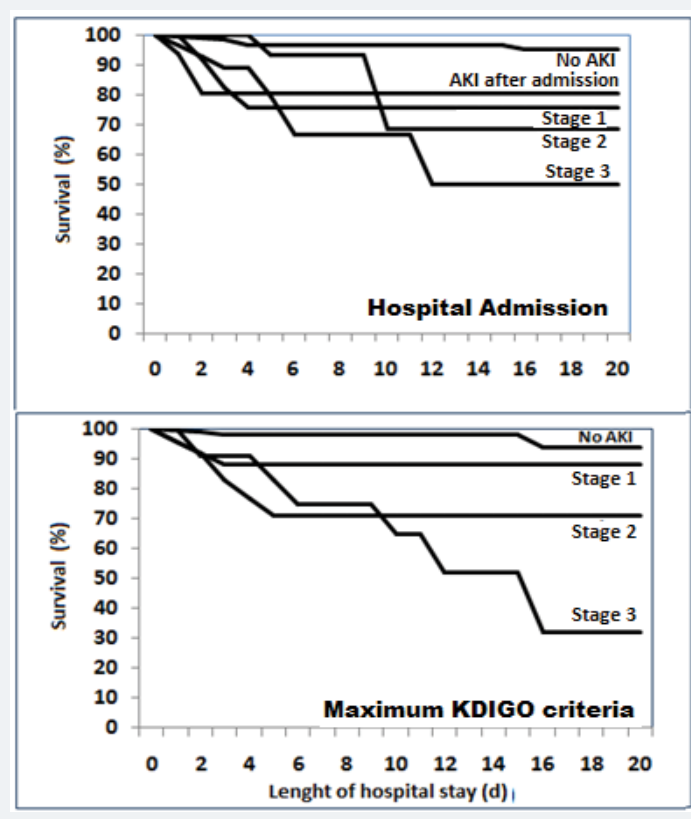

Figure 3: Kaplan-Meier curves for patient survival by admission and maximum KDIGO criteria. Patients discharged alive were censored. Log-rank test: $x^{2}=32.54 ; p<0.0001$.

\section{Discussion}

We conducted a retrospective study of hospitalized patients and we analyzed the ability of the KDIGO criteria to predict renal outcomes, need for RRT, length of hospital stay, and hospital mortality in patients with malaria associated AKI. The KDIGO criteria represents a new classification system [7], and recently published studies have shown that KDIGO criteria provided a well-balanced instrument for the determination of occurrence rates and/or outcomes of patients with AKI [13-16]. We found that AKI, defined by the KDIGO criteria, had a high overall incidence in 706 patients with falciparum malaria infection. Prior to hospital admission, $83.7 \%$ of the 86 patients already had AKI, with only 14 patients of the total population developing AKI after admission to hospital. In addition, another 18 patients with malarial AKI at admission experienced worsening of their KDIGO statuses during the hospitalization period.

We confirmed that the new KDIGO criteria show robust predictive abilities for disease severity and hospital outcomes in this large cohort of patients with malaria and AKI, as was similarly shown in recent studies in a variety of critically ill patients [16-20]. Upon hospital admission, no patients with AKI Stage 1 required for renal replacement therapy, while dialysis was required in $23 \%$ and $42 \%$ of the patients with AKI Stage 2 and 3, respectively. In addition, no patients with maximum AKI Stage 1 during hospitalization required hemodialysis. Furthermore, length of hospital stay and mortality risk increased along with the severity of the AKI.

The new KDIGO criteria could provide a simple definition of AKI for patients with malaria, and our data suggest that it provided a well-balanced classification system for the determination of patients with different levels of severity of AKI, at least insofar as risk of renal replacement therapy requirement, length of hospital stay, or hospital mortality in malarial AKI was concerned. In a previous analysis of AKI in these patients with malaria, we reported that RIFLE criteria were useful and accurate in predicting overall outcomes for patients with malarial AKI [21].

These findings are of great importance for patients with more severe malarial AKI, especially in developing countries at which an early access to a tertiary care center, where prompt ICU supportive management and dialysis can be instituted, is very limited [15-18]. 
Several limitations of our study deserve to be considered. As a single-center study from a general hospital in the northeast Southern Africa, generalizability is limited. Incidence estimates, length of hospital stay, mortality rates, and concomitant diagnoses vary across hospital types and regions, although our estimates for AKI are in keeping with others from geographically disparate regions. We did not have the baseline serum creatinine or the hourly urine output data for all patients. Finally, as our outcome data were evaluated at the time of discharge, we may have underestimated the mortality rate of patients with AKI. Overall, we recognize that any biases would influence the effectiveness of the KDIGO criteria and thus not significantly influence our conclusions. Long-term mortality, chronic kidney disease (CKD) development, and CKD progression were not evaluated in this study but are important areas for future research

In conclusion, we performed a relatively large single-center study of the KDIGO criteria for AKI in hospitalized patients with falciparum malaria. The KDIGO criteria identified and classified an estimated $12.5 \%$ of all the patients with malaria diagnoses as also having AKI. The KDIGO criteria were robust in this population for predicting the need for dialysis, length of hospital stay, and overall hospital mortality. The results of the present study support the utilization of the KDIGO criteria in predicting outcomes for patients with malarial AKI.

\section{References}

1. Muentener $P$, Schlagenhauf $P$, Steffen R. Imported malaria (1985-95): trends and perspectives. Bull World Health Organ. 1999; 77: 560-566. Ref.: https://goo.gl/mvdhAr

2. Sachs J, Malaney P. The economic and social burden of malaria. Nature. 2002; 415: 680-685. Ref.: https://goo.gl/9oV4kb

3. Das BS. Renal failure in malaria. J Vector Borne Dis. 2008; 45: 83-97. Ref.: https://goo.gl/vRskvS

4. Sitprija V. Nephropathy in falciparum malaria. Kidney Int. 1988; 34: 867-877. Ref.: https://goo.gl/4pG4bG

5. Bulbol WS, Carneiro M, Cardoso AC, Alecrim WD, de Albuquerque BC, et al. Acute kidney failure in Plasmodium falciparum malaria. Rev Inst Med Trop Sao Paulo. 1982; 24: 58-61. Ref.: https://goo.gl/LJSGim

6. Koopmans LC, van Wolfswinkel ME, Hesselink DA, Hoorn EJ, Koelewijn R, et al. Acute kidney injury in imported Plasmodium falciparum malaria. Malar J. 2015; 24; 14:523. Ref.: https://goo.gl/fC1dPV

7. Kidney Disease: Improving Global Outcomes (KDIGO) Acute Kidney Injury Work Group KDIGO Clinical Practice Guideline for Acute Kidney Injury. Kidney Int Suppl. 2012; 2: 1-138.

8. Bellomo R, Ronco C, Kellum JA, Mehta RL, Palevsky P, et al. Acute renal failure - definition, outcome measures, animal models, fluid therapy and information technology needs: The second international consensus conference of the acute dialysis quality initiative (ADQI) group. Crit Care. 2004; 8: 204212. Ref.: https://goo.gl/keemN5

9. Molitoris BA, Levin A, Warnock DG, Joannidis M, Mehta RL, et al. Improving outcomes from acute kidney injury. J Am Soc Nephrol. 2007; 18: 1992-1994. Ref.: https://goo.gl/vPD4Xk

10. Pereira M, Rodrigues N, Godinho I, Gameiro J, Neves M, et al. Acute kidney injury in patients with severe sepsis or septic shock: a comparison between the 'Risk, Injury, Failure, Loss of kidney function, End-stage kidney disease' (RIFLE), Acute Kidney Injury Network (AKIN) and Kidney Disease: Improving Global Outcomes (KDIGO) classifications. Clin Kidney J. 2017; 10: 332-340. Ref.: https://goo.gl/W764Df

11. Abosaif NY, Tolba YA, Heap M, Russell J, El Nahas AM. The outcome of acute renal failure in the intensive care unit according to RIFLE: model application, sensitivity, and predictability. Am J Kidney Dis. 2005; 46: 1038-1048. Ref.: https://goo.gl/4L3U9R

12. Daily JP. Malaria 2017 Update on the Clinical Literature and Management. Curr Infect Dis Rep. 2017 19: 28. Ref.: https://goo.gl/L8sucE

13. Nisula S, Kaukonen KM, Vaara ST, Korhonen AM, Poukkanen M, et al. Incidence, risk factors and 90day mortality of patients with acute kidney injury in Finnish intensive care units: the FINNAKI study. Intensive Care Med. 2013; 39: 420-428. Ref.: https://goo.gl/CENYQ5 
14. Roy AK, Mc Gorrian C, Treacy C, Kavanaugh E, Brennan A, et al. A Comparison of Traditional and Novel Definitions (RIFLE, AKIN, and KDIGO) of Acute Kidney Injury for the Prediction of Outcomes in Acute Decompensated Heart Failure. Cardiorenal Med 2013; 3: 26-37. Ref.: https://goo.gl/JPqvyZ

15. Fujii T, Uchino S, Takinami M, Bellomo R. Validation of the Kidney Disease Improving Global Outcomes criteria for AKI and comparison of three criteria in hospitalized patients. Clin J Am Soc Nephrol. 2014; 9: 848-854. Ref.: https://goo.gl/YyWXKU

16. Zeng X, McMahon GM, Brunelli SM, Bates DW, Waikar SS. Incidence, outcomes, and comparisons across definitions of AKI in hospitalized individuals. Clin J Am Soc Nephrol. 2014; 9: 12-20. Ref.: https://goo.gl/1L6UZW

17. Ricci Z, Cruz DN, Ronco C: Classification and staging of acute kidney injury: beyond the RIFLE and AKIN criteria. Nat Rev Nephrol. 2011; 7: 201-208. Ref.: https://goo.gl/gtKQ16

18. Bhadade R, De'Souza R, Harde MJ, Mehta KS, Bhargava P. A Prospective Study of Acute Kidney Injury According to KDIGO Definition and its Mortality Predictors. J Assoc Physicians India. 2016; 64: 22 28. Ref.: https://goo.gl/uPK9H2

19. Bouchard J, Mehta RL. Acute Kidney Injury in Western Countries. Kidney Dis (Basel). 2016; 2: 103110. Ref.: https://goo.gl/ixmCGJ

20. Li PK, Burdmann EA, Mehta RL. World Kidney Day Steering Committee 2013. Acute kidney injury: global health alert. Kidney Int. 2013; 83: 372-376. Ref.: https://goo.gl/Ff989X

21. Brandao J, Romao Jr JE. Acute kidney injury in patients with falciparum malaria. An assessment of the RIFLE criteria. Nephrol Dial Transplant. 2012; 27: 21-22. 